\title{
Non-Asymptotic Connectivity of Random Graphs and Their Unions
}

\author{
Beth Bjorkman* Matthew Hale ${ }^{\dagger}$ Thomas Lamkin ${ }^{\ddagger}$ Benjamin Robinson** Craig Thompson $^{\dagger \dagger}$
}

\begin{abstract}
Graph-theoretic methods have seen wide use throughout the literature on multi-agent control and optimization. When communications are intermittent and unpredictable, such networks have been modeled using random communication graphs. When graphs are time-varying, it is common to assume that their unions are connected over time, yet, to the best of our knowledge, there are not results that determine the number of finite-size random graphs needed to attain a connected union. Therefore, this paper bounds the probability that individual random graphs are connected and bounds the same probability for connectedness of unions of random graphs. The random graph model used is a generalization of the classic Erdős-Rényi model which allows some edges never to appear. Numerical results are presented to illustrate the analytical developments made.
\end{abstract}

\section{INTRODUCTION}

Multi-agent systems have been studied in a number of applications, including sensor networks [11], communications [21], and smart power grids [6]. Across these applications, the agents in a network and their associated communications are often abstractly represented as graphs [26]. In general, graph-theoretic methods in multi-agent systems represent each agent as a node in a graph and each communication link as an edge, and multi-agent coordination algorithms have been developed for both static and time-varying graphs [26, Chapter 1.4].

Time-varying random graphs in particular have been used to model communications which are unreliable and intermittent due to interference and poor channel quality [26, Chapter 5]. Such graphs have seen use in a number of multi-agent settings. For example, distributed agreement problems over random graphs are studied in [37] and [19], while optimization over random graphs was explored in [25]. The work in [43] provides a means to modify random graphs to make them robust to network failures, and [24] discusses general properties of random graphs as they pertain to multi-agent systems. A broad survey of graph-theoretic results for control can be found in [26], and well-known graph-theoretic results in optimization include [3], [29], [44].

When time-varying graphs (random or not) are used, a common assumption is that the unions of these graphs are connected over intervals of some finite length, i.e., the graph containing all edges present over time is itself a

\footnotetext{
* Iowa State University Department of Mathematics, bjorkman@iastate.edu

${ }^{\dagger}$ Department of Mechanical and Aerospace Engineering, University of Florida, matthewhale@ufl.edu

${ }^{\ddagger}$ lamkintd@ miamioh.edu

**Air Force Research Laboratory Sensors Directorate

${ }^{\dagger}$ University of Arizona Program in Applied Mathematics, craigthompson@math.arizona.edu
} 
connected graph. A partial sampling of works using this assumption (or a related variant) includes [27], [34], [40], [39], [35], [8], [22], [3], [20], [36], [30], [29], [31], [44], [15], [28]. In addition, some works derive convergence rates or other results that explicitly use the length of such intervals, including [15], [40], [28], [39], [8], [3], [36], [31], [29]. In applying these results, one may wish to determine the time needed for the system to attain a connected union graph. To the best of our knowledge, no study has been undertaken that addresses this problem for unions of finite-size random graphs, despite their frequent use in multi-agent systems.

Owing to the success of Erdős-Rényi graphs in modeling some time-varying multi-agent communications [26. Chapter 5], we consider unions of random graphs generated by a generalization of the Erdős-Rényi model. In particular, we examine the connectedness of such graphs and their unions. Formally, this paper first lower-bounds the number of graphs needed to make their "expected union" connected (in a precise sense). It then finds a lower bound for the probability that a union of random graphs is connected as a function of graph model parameters.

Our results use spectral properties of the Laplacian of a union of random graphs. We utilize the first order statistic of the set of non-trivial eigenvalues to bound the expected value of the Laplacian's second-smallest eigenvalue, called the algebraic connectivity [16] of the underlying union graph. This bound in turn enables a lower bound on the probability of the algebraic connectivity exceeding some given threshold.

Random graphs' Laplacians are random matrices, and thus our approach relies on the spectral properties of random matrices of a particular form. One common approach to analyzing the spectra of random matrices is to let the dimension of the matrix grow arbitrarily large [12], [17], [42]; the work in [9] considers similar asymptotic results focused specifically on Laplacians of random graphs. For random graphs, a common approach is to derive results in which the size of the graph grows arbitrarily large, and doing so enables results that hold for almost all graphs [5]. In particular, seminal work by Erdős and Rényi showed that an Erdős-Rényi graph on $n$ nodes with edge probability $p$ is almost surely connected as $n \rightarrow \infty$ if $p \geq c \frac{\log n}{n}$ and $c>1$. While there is clear theoretical appeal to such results, our focus on multi-agent systems leads us to consider non-asymptotic results precisely because such systems are very often comprised by a fixed, finite number of agents. We therefore study and characterize random graphs with a fixed, finite number of nodes, i.e., with $n$ fixed, and we will develop a novel statistical approach to characterize such graphs.

In addition, while references [13] and [14], and many other works on random graphs consider edge probabilities that bear some known relationship to the number of nodes in a graph [23], [38], we do not do so here. The use of random graphs to model multi-agent communications is inspired by applications in which poor channel quality, interference, and other factors make communications unreliable. In such cases, the probability of a communication link being active may not bear any known relationship to the size of the network. Accordingly, we proceed with edge probabilities and network sizes that are fixed and not assumed to be related. In particular, we require only $p \in(0,1)$ and avoid any assumption that $p \sim \frac{\log n}{n}$ or that $p$ is any known function of $n$, in contrast to existing results such as [14] and [5].

Related work in [2] explores the properties of isoperimetric constants of random graphs, which are known to be related to algebraic connectivity via Cheeger's Inequality [7]. However, the random graph model in [2] always adds one new edge at every time step, while the unions of random graphs we consider can add zero, one, or many new 
edges at every time step. Accordingly, the conclusions reached in [2] do not apply to the random graph model we consider. Developments in [10] assess connectivity of the Erdős-Rényi random graph model through analyzing their cover times. However, the results in [10] are asymptotic, in that they consider $n \rightarrow \infty$, and thus their conclusions do not readily apply to the finite-sized graphs we study.

Additional work in [33, Section V] studies eigenvalues of Laplacians of Erdős-Rényi graphs as we do in this work. In particular, results in that section study the algebraic connectivity of Erdős-Rényi graphs, though they do so in the asymptotic regime (i.e., as $n \rightarrow \infty$ ). Our results differ from that work because they apply for arbitrary, fixed values of $n$, rather than to the case in which $n \rightarrow \infty$.

The rest of the paper is organized as follows. Section $\Pi$ reviews the required elements of graph theory and gives a formal statement for the problem that is the focus of this paper. Section [II] computes the first order statistic of the eigenvalues of random graph Laplacians and certain statistical properties of the eigenvalues to enable the results of Section IV Section IV then presents the main results of the paper and solves the problem stated in Section $\Pi$ Section $\mathrm{V}$ next provides numerical solutions to several instantiations of the problem studied. Finally, Section VI provides concluding remarks.

\section{ReView of GRAPH Theory AND PRoblem Statement}

In this section, we review the required elements of graph theory. We begin with basic definitions, including the definition of algebraic connectivity, and then review the Erdős-Rényi model for random graphs and introduce a generalization that we use. Throughout this paper, all uses of the phrase "random graphs" refer to Erdős-Rényi-type graphs. Then we formally state the statistical graph connectivity problem solved in this paper. Below, we use the notation $[n]:=\{1, \ldots, n\}$ for any $n \in \mathbb{N}$ and $|\cdot|$ to represent the cardinality of a set.

\section{A. Basic Graph Theory}

A graph $G=(V, E)$ is defined over a set of nodes, denoted $V$, and describes connections between these nodes in the form of edges, which are contained in an edge set $E$. For $n$ nodes, $n \in \mathbb{N}$, the elements of $V$ are indexed over $[n]$. The set of edges in the graph is a subset $E \subseteq V \times V$, where a pair $(i, j) \in E$ if nodes $i$ and $j$ share a connection, and $(i, j) \notin E$ if they do not. This paper considers graphs which are undirected, meaning an edge $(i, j)$ is not distinguished from an edge $(j, i)$, and simple, so that $(i, i) \notin E$ for all $i$. The degree of node $i \in V$ is defined as

$$
d_{i}=|\{j \in V \mid(i, j) \in E\}|,
$$

which is equal to the number of vertices sharing an edge with vertex $i$. One main focus of this paper is connected graphs.

Definition 2.1 (E.g., [18]): A graph $G$ is called connected if for all $i \in[n]$ and $j \in[n], i \neq j$, there is a sequence of edges one can traverse from node $i$ to node $j$, i.e., there is a sequence of vertices $\left\{i_{\ell}\right\}_{\ell=1}^{k}$ such that $E$ contains all of the edges

$$
\left(i, i_{1}\right),\left(i_{1}, i_{2}\right),\left(i_{2}, i_{3}\right), \ldots,\left(i_{k-1}, i_{k}\right),\left(i_{k}, j\right)
$$


The results of this paper are developed in terms of graph Laplacians, which are defined in terms of the adjacency and degree matrices of a graph. The adjacency matrix $A(G) \in \mathbb{R}^{n \times n}$ associated with the graph $G$ is defined element-wise as

$$
(A(G))_{i j}=\left\{\begin{array}{ll}
1 & (i, j) \in E \\
0 & \text { otherwise }
\end{array} .\right.
$$

When there is no ambiguity, we will simply denote $A(G)$ by $A$. Because we consider undirected graphs, $A$ is symmetric by definition. The degree matrix $D(G) \in \mathbb{R}^{n \times n}$ associated with a graph $G$ is the diagonal matrix $D(G)=\operatorname{diag}\left(d_{1}, d_{2}, \ldots, d_{n}\right)$, which we will denote $D$ when $G$ is clear from context. By definition, $D$ is also symmetric. The Laplacian of a graph $G$ is then defined as $L(G)=D(G)-A(G)$, which will be written simply as $L$ when $G$ is unambiguous.

The results of this paper rely in particular on spectral properties of $L$. Letting $\lambda_{k}(\cdot)$ denote the $k^{\text {th }}$ smallest eigenvalue of a matrix, it is known that $\lambda_{1}(L)=0$ for all graph Laplacians [26], and thus we have

$$
0=\lambda_{1}(L) \leq \lambda_{2}(L) \leq \cdots \leq \lambda_{n}(L)
$$

The value of $\lambda_{2}(L)$ is central to the work in this paper and some other works in graph theory, and it gives rise to the following definition.

Definition 2.2 ([16]): The algebraic connectivity of a graph $G$ is the second smallest eigenvalue of its Laplacian, $\lambda_{2}(L)$, and $G$ is connected if and only if $\lambda_{2}(L)>0$.

This paper is dedicated to studying the statistical properties of $\lambda_{2}=\lambda_{2}(L)$ for random graphs and unions of random graphs. Toward doing so, we now review the basics of the random graph model we study.

\section{B. Random Graphs}

Several well-known random graph models exist in the literature [13], [41], and Erdôs-Rényi graphs in particular have been successfully used in the multi-agent systems literature. Erdős-Rényi graphs can model, for example, unreliable, intermittent and time-varying communications in multi-agent networks [26], and we therefore consider the Erdős-Rényi model in this paper. Under this model, a graph on $n$ vertices contains each admissible edge with some fixed edge probability $p \in(0,1)$. Therefore, for each $i \in[n]$ and $j \in[n]$ with $i \neq j$, an Erdős-Rényi graph satisfies

$$
\mathbb{P}[(i, j) \in E]=p \text { and } \mathbb{P}[(i, j) \notin E]=1-p .
$$

We denote the sample space of all Erdős-Rényi graphs on $n$ nodes with edge probability $p$ by $\mathcal{G}(n, p)$, and we denote the set of Laplacians of all such graphs by $\mathcal{L}(n, p)$. We will primarily study the following variant of the Erdôs-Rényi model, which contains the typical Erdős-Rényi formulation as a special case. 
Definition 2.3: Let $G=(V(G), E(G))$ be a connected graph on $n$ vertices. An Erdös-Rényi graph corresponding to $G$ is a random graph on $n$ vertices with an edge set $E$ which satisfies

$$
\mathbb{P}[(i, j) \in E]=\left\{\begin{array}{ll}
p & (i, j) \in E(G) \\
0 & (i, j) \notin E(G)
\end{array} .\right.
$$

Correspondingly,

$$
\mathbb{P}[(i, j) \notin E]=\left\{\begin{array}{rl}
1-p & (i, j) \in E(G) \\
1 & (i, j) \notin E(G)
\end{array} .\right.
$$

We denote the sample space of all Erdős-Rényi graphs corresponding to $G$ by $\mathcal{G}(G, p)$. We note that $\mathcal{G}(n, p)=$ $\mathcal{G}\left(K_{n}, p\right)$, where $K_{n}$ represents the complete graph on $n$ vertices.

Some well-known results in the graph theory literature assume that $p$ has some known relationship to $n$ [4], or else that the number of edges in a random graph has some relationship to the number of nodes in the graph [14]. While the theoretical utility of these relationships is certainly clear from those works, these relationships will often not hold in multi-agent systems simply because their communications are affected by a wide variety of external factors. We therefore proceed with a value of $p \in(0,1)$ that is not assumed to have any relationship to the value of $n$. In the study of multi-agent systems, it is also common for algorithms and results to be stated in terms of unions of graphs, which we define now.

Definition 2.4: For a collection of graphs $\left\{G_{k}=\left(V, E_{k}\right)\right\}_{k=1}^{T}$ defined on the same node set $V$, the union of these graphs, denoted $U_{T}$, is defined as $U_{T}:=\cup_{k=1}^{T} G_{k}=\left(V, \cup_{k=1}^{T} E_{k}\right)$, i.e., the union graph $U_{T}$ contains all edges from all $T$ graphs in the union.

\section{Problem Statement}

A common requirement in some multi-agent systems is that the communication graphs in a network form a connected union graph over intervals of some fixed length. To help determine when this occurs with random interactions, we solve the following problem in this paper.

Problem 1: Given a connected graph $G$ on a fixed, finite number of nodes $n \in \mathbb{N}$ and a fixed, arbitrary probability $p \in(0,1)$, find a lower bound for the probability that a given graph $\hat{G} \in \mathcal{G}(G, p)$ is connected. That is, find a lower bound for $\mathbb{P}\left[\lambda_{2}(\hat{G})>0\right]$.

While this problem is defined in terms of a single random graph, we can use it to derive results for a union of random graphs because such unions are themselves equivalent to single random graphs, albeit with a different edge probability.

Lemma 2.5: Let $\mathcal{U}_{T}(G, p)$ denote the set of all unions of $T$ random graphs on $n$ nodes with edge probability $p$, i.e., $\mathcal{U}_{T}(G, p):=\left\{\cup_{k=1}^{T} G_{k} \mid G_{k} \in \mathcal{G}(G, p)\right\}$. Then

$$
\mathcal{U}_{T}(G, p)=\mathcal{G}\left(G, 1-(1-p)^{T}\right) .
$$

Proof: Fix any edge $(i, j) \in E(G)$. Then the edge $(i, j)$ is absent in $\mathcal{U}_{T}(G, p)$ only if it is absent in all $T$ graphs that comprise $\mathcal{U}_{T}(G, p)$. Observe that the edge between $i$ and $j$ is absent in each $G_{k}$ with probability $(1-p)^{T}$. Then edge $(i, j)$ is present in $\mathcal{U}_{T}(G, p)$ with probability $1-(1-p)^{T}$. 
With Lemma 2.5, results pertaining to individual random graphs can easily be applied to unions of such graphs.

\section{Differences with Asymptotic Analyses}

In this subsection, we highlight the differences between what is asked in Problem 1 and classical results in the study of random graphs. In particular, the asymptotic connectivity of Erdős-Rényi graphs is well-known. To highlight the key differences with that result, we reproduce it here.

Proposition 1 (From [14]): A graph $G \in \mathcal{G}(n, p)$ is almost surely connected if $p \geq c \frac{\log n}{n}$ and $c>1$. Explicitly,

$$
\lim _{n \rightarrow \infty} \mathbb{P}[G \in \mathcal{G}(n, p) \text { is connected }]=1
$$

if $p=c \frac{\log n}{n}$ and $c>1$.

The notion of "almost surely" used in the statement of Proposition 1 dates back to seminal work of Erdôs and Rényi and refers to properties that hold "with probability tending to 1 for $n \rightarrow+\infty$ " [14, Page 3]. That is, drawing conclusions about random graphs that hold "almost surely" requires taking the limit as $n \rightarrow \infty$. This notion is sometimes called "asymptotically almost surely", e.g., in [33], to reflect the inherently asymptotic nature of its conclusions.

The focus on asymptotics is the first essential difference between what Problem 1 seeks to solve and existing results: Problem 1 considers networks of a fixed but arbitrary number of nodes $n \in \mathbb{N}$, while Proposition 1 assesses connectivity as $n \rightarrow \infty$. The finiteness of $n$ in Problem 1 precludes the use of Proposition 1 to solve it. The second key difference is that Problem 1 does not permit the assumption that $p$ is a specified function of $n$. Instead, in Problem $1, p \in(0,1)$ is fixed but arbitrary. Thus, any connectivity conclusions drawn from Proposition 1 are necessarily inapplicable to Problem 1.

We illustrate the need for new techniques to solve Problem 1 with an example below. In it, we show that the setting of Problem 1 requires us to consider graphs that are neither connected with probability 1 , nor disconnected with probability 1.

Example 1: Fix $n=3$ nodes and consider the underlying graph $G=K_{3}$, i.e., the complete graph on 3 nodes. Set $p=\frac{1}{2}=1.365 \frac{\log 3}{3}$. If we take $n \rightarrow \infty$, then this choice of $p$ satisfies the condition for connectivity in Proposition 1, but the probability that a graph in $\mathcal{G}\left(3, \frac{1}{2}\right)$ is connected is not near 1 , as follows. We consider graphs in $\mathcal{G}\left(K_{3}, \frac{1}{2}\right)=\mathcal{G}\left(3, \frac{1}{2}\right)$, which are classical Erdős-Rényi graphs. With $n=3$ fixed, there are eight possible graphs in $\mathcal{G}\left(3, \frac{1}{2}\right)$, shown in Figure 1

Graphs $G_{1}, G_{2}, G_{3}$, and $G_{4}$ are not connected, while graphs $G_{5}, G_{6}, G_{7}$, and $G_{8}$ are connected. Because $p=\frac{1}{2}$, all eight graphs are equiprobable and thus

$$
\mathbb{P}\left[G \in \mathcal{G}\left(3, \frac{1}{2}\right) \text { is connected }\right]=\frac{1}{2} .
$$

Then a random graph $G \in \mathcal{G}\left(3, \frac{1}{2}\right)$ is neither connected nor disconnected with probability 1 .

In Example 1, the lack of either connectedness or disconnectedness with probability 1 is not captured by classical results such as Proposition 1 and new analyses are required to account for and quantify this behavior. That is the subject of this paper.

Section III next provides theoretical developments that will enable the solution to Problem 1 in Section IV 


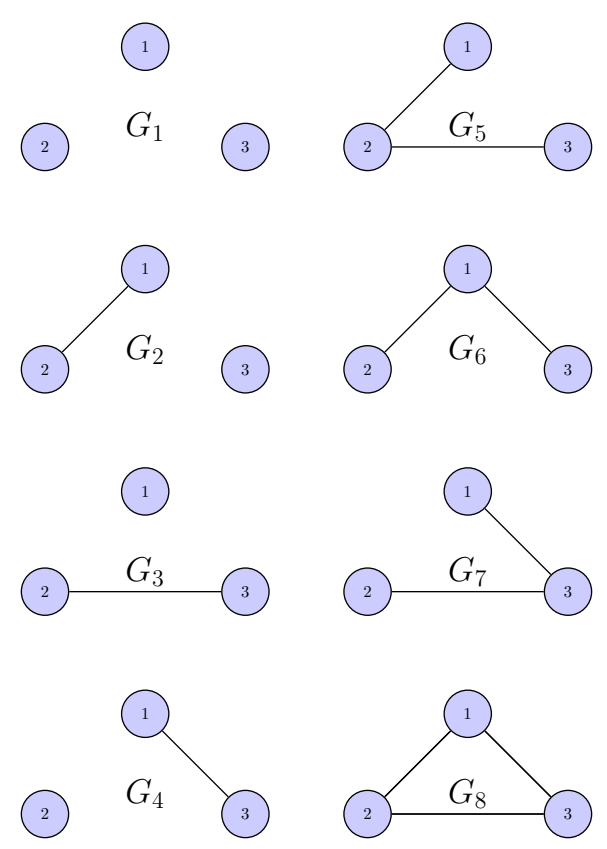

Fig. 1. The set of all eight graphs in $\mathcal{G}\left(K_{3}, \frac{1}{2}\right)=\mathcal{G}\left(3, \frac{1}{2}\right)$. All graphs are equiprobable because $p=\frac{1}{2}$, and thus half of all graphs in $\mathcal{G}\left(3, \frac{1}{2}\right)$ are connected.

\section{ORder Statistics over RAndom Eigenvalues}

Solving Problem 1 will require bounds on the expected algebraic connectivity of a random graph of interest. This section derives such bounds in terms of known quantities by relating the expected algebraic connectivity of a random graph to other spectral properties.

Let $G$ be a connected graph with $m$ edges and $n$ vertices labeled by $[n]$. Let $d_{i}$ be the degree of vertex $i$ in $G$. Let $\hat{G} \in \mathcal{G}(G, p)$ be a random graph with Laplacian $L$ whose eigenvalues are denoted $\lambda_{1}(\hat{G})$ through $\lambda_{n}(\hat{G})$. Let $\ell$ be a random variable equal to $\lambda_{i}$ with probability $\frac{1}{n-1}$ for $i \geq 2$. Take $N$ independent samples of $\ell$, denoted $\ell_{1}, \ldots, \ell_{N}$, and place the results in ascending order to create the list

$$
\ell_{1: N}, \ell_{2: N}, \ldots, \ell_{N: N}
$$

where $\ell_{j: N}$ denotes the $j^{\text {th }}$ largest sample out of the $N$ total samples. Then $\ell_{1: N}$ is by definition the first order statistic for the samples $\left\{\ell_{j}\right\}_{j=1}^{N}$. Analyzing these order statistics for $\hat{G}$, we obtain the following relationship to the expected value of $\lambda_{2}(\hat{G})$.

Lemma 3.1: Consider a connected graph $G$ with $m$ edges and vertex set indexed over $[n]$. For $\hat{G} \in \mathcal{G}(G, p)$ we have

$$
\mathbb{E}\left[\ell_{1: N}\right] \leq \mathbb{E}\left[\lambda_{2}(\hat{G})\right]\left(1-\left(\frac{n-2}{n-1}\right)^{N-1}\right)+\frac{2 m p}{n-2}\left(\frac{n-2}{n-1}\right)^{N}
$$


Proof: Let $r=\frac{1}{n-1}$. Then

$$
\begin{aligned}
\mathbb{E}\left[\ell_{1: N}\right] & =\mathbb{E}\left[\ell_{1: N} \mid \exists k: \ell_{k}=\lambda_{2}\right] \mathbb{P}\left[\exists k: \ell_{k}=\lambda_{2}\right]+\mathbb{E}\left[\ell_{1: N} \mid \nexists k: \ell_{k}=\lambda_{2}\right] \mathbb{P}\left[\nexists k: \ell_{k}=\lambda_{2}\right] \\
& =\mathbb{E}\left[\ell_{1: N} \mid \exists k: \ell_{k}=\lambda_{2}\right]\left(1-(1-r)^{N}\right)+\mathbb{E}\left[\ell_{1: N} \mid \nexists k: \ell_{k} \neq \lambda_{2}\right](1-r)^{N} .
\end{aligned}
$$

Observe that

$$
\mathbb{E}\left[\ell_{1: N} \mid \exists k: \ell_{k}=\lambda_{2}\right]=\mathbb{E}\left[\lambda_{2}\right]
$$

Furthermore, by definition we have

$$
\begin{aligned}
\mathbb{E}\left[\ell_{1: N} \mid \nexists k: \ell_{k} \neq \lambda_{2}\right] & :=\mathbb{E}\left[\min _{j \in[N]} \ell_{j} \mid \nexists k: \ell_{k} \neq \lambda_{2}\right] \\
& \leq \mathbb{E}\left[\ell_{j}, j \text { arbitrary } \mid \nexists k: \ell_{k} \neq \lambda_{2}\right] \\
& =\frac{1}{n-2} \sum_{i=3}^{n} \mathbb{E}\left[\lambda_{i}\right] \\
& =\frac{1}{n-2}\left(\mathbb{E}[\operatorname{tr} L]-\mathbb{E}\left[\lambda_{2}\right]\right),
\end{aligned}
$$

where the last line follows from the fact that $\mathbb{E}\left[\lambda_{1}\right]=0$ for all graphs $G$.

Substituting Equations (2) and (3) into Equation (1) gives

$$
\mathbb{E}\left[\ell_{1: N}\right] \leq \mathbb{E}\left[\lambda_{2}\right]\left(1-(1-r)^{N}\right)+\left(\frac{\mathbb{E}[\operatorname{tr} L]-\mathbb{E}\left[\lambda_{2}\right]}{n-2}\right)(1-r)^{N} .
$$

Next, we note that $\mathbb{E}[\operatorname{tr} L]=\sum_{i=1}^{n} p d_{i}$ by construction of the Laplacian. Then we have

$$
\mathbb{E}\left[\ell_{1: N}\right] \leq \mathbb{E}\left[\lambda_{2}\right]\left(1-(1-r)^{N}-\frac{1}{n-2}(1-r)^{N}\right)+\frac{\sum_{i=1}^{n} p d_{i}}{n-2}(1-r)^{N}
$$

and the lemma follows by factoring and the Handshake Lemma $\left(\sum_{i=1}^{n} d_{i}=2 m\right)$.

Having put $\mathbb{E}\left[\lambda_{2}(\hat{G})\right]$ in terms of $\mathbb{E}\left[\ell_{1: N}\right]$, we next focus on bounding $\mathbb{E}\left[\ell_{1: N}\right]$ in terms of known quantities. Toward doing so, we state the following lemma.

Lemma 3.2 (Equation (4), [17): Let $X_{1}, X_{2}, \ldots, X_{m}$ be jointly distributed with common mean $\mu$ and variance $\sigma^{2}$. Then the $k^{t h}$ order statistic of this collection, denoted $X_{k: m}$, has expectation bounded according to

$$
\mu-\sigma \sqrt{\frac{m-k}{k}} \leq \mathbb{E}\left[X_{k: m}\right] .
$$

The bound in Lemma 3.2 was shown in [1] to be tight for collections of random variables with identical means and variances. Because each $\ell_{k}$ has the same mean and variance, Lemma 3.2 is an attractive choice in computing the required bound on $\mathbb{E}\left[\ell_{1: N}\right]$. Therefore, we now work to apply Lemma 3.2 to $\ell_{1: N}$.

Lemma 3.3: Consider a connected graph $G=(V, E)$ on $n$ vertices with $m$ edges so that vertex $i$ has degree $d_{i}$, and suppose that $\left\{\ell_{k}\right\}_{k \in[N]}$ are as before. Then, for $\hat{G} \in \mathcal{G}(G, p)$, we find that each $\ell_{k}$ has mean $\mu$ and variance $\sigma^{2}$ given by

$$
\mu=\frac{2 m p}{n-1}
$$

and

$$
\sigma^{2}=\frac{p}{(n-1)^{2}}\left((n-1)\left(2 m(2-p)+p \sum_{i=1}^{n} d_{i}^{2}\right)-4 m^{2} p\right) .
$$


Proof: Observe that

$$
\mu=\mathbb{E}\left[\frac{1}{n-1} \operatorname{tr}(L)\right]=\frac{2 m p}{n-1} .
$$

Next, set $r=\frac{1}{n-1}$. Let $L$ be the Laplacian of $\hat{G}$, and denote its $i^{t h} j^{t h}$ off-diagonal entry by $X_{i j}$. If $(i, j) \notin E$, then $X_{i j}=0$. If $(i, j) \in E$, then

$$
X_{i j}=\left\{\begin{array}{ll}
1 & \text { with probability } p \\
0 & \text { with probability } 1-p
\end{array},\right.
$$

i.e., $X_{i j}$ is a Bernoulli random variable when $(i, j) \in E$. Then $X_{i i}=\sum_{1 \leq j \leq n, j \neq i} X_{i j}$. By definition we have

$$
\begin{aligned}
\sigma^{2} & =\mathbb{E}\left[\ell_{k}^{2}\right]-\mathbb{E}\left[\ell_{k}\right]^{2} \\
& =\frac{1}{n-1} \operatorname{tr}\left(\mathbb{E}\left[L^{2}\right]\right)-\left(4 m^{2} p^{2}\right)\left(\frac{1}{n-1}\right)^{2} \\
& =r \sum_{i=1}^{n} \mathbb{E}\left[\left(L^{2}\right)_{i i}\right]-\left(4 m^{2} p^{2}\right) r^{2},
\end{aligned}
$$

where the second equation follows from the value of $\mu$ and the fact that the eigenvalues of $L^{2}$ are the squares of the eigenvalues of $L$.

We then expand to find

$$
\begin{aligned}
\sigma^{2} & =r \sum_{i=1}^{n}\left(\mathbb{E}\left[\left(\sum_{j=1, j \neq i}^{n} X_{i j}\right)^{2}\right]+\sum_{j=1, j \neq i}^{n} \mathbb{E}\left[X_{i j}^{2}\right]\right)-\left(4 m^{2} p^{2}\right) r^{2} \\
& =r \sum_{i=1}^{n}\left(p d_{i}+d_{i}\left(d_{i}-1\right) p^{2}+p d_{i}\right)-\left(4 m^{2} p^{2}\right) r^{2},
\end{aligned}
$$

which follows from the independence of $X_{i j}$ and $X_{i k}$ (because edges are independent). Re-arranging terms, we find

$$
\begin{aligned}
\sigma^{2} & =r\left(2 p \sum_{i=1}^{n} d_{i}+p^{2} \sum_{i=1}^{n} d_{i}^{2}-p^{2} \sum_{i=1}^{n} d_{i}\right)-4 m^{2} p^{2} r^{2} \\
& =r\left(4 m p+p^{2} \sum_{i=1}^{n} d_{i}^{2}-2 p^{2} m\right)-4 m^{2} p^{2} r^{2} \\
& =\frac{p}{(n-1)^{2}}\left((n-1)\left(2 m(2-p)+p \sum_{i=1}^{n} d_{i}^{2}\right)-4 m^{2} p\right),
\end{aligned}
$$

which follows from the Handshake Lemma.

With $m, n \in \mathbb{N}, \underline{d}=\left(d_{1}, d_{2}, \ldots d_{n}\right)$, and $0 \leq p \leq 1$, we define

$$
S(m, n, p, \underline{d})=\left(2 m p(n-1)(2-p)+p^{2}(n-1) \sum_{i=1}^{n} d_{i}^{2}-4 m^{2} p^{2}\right)^{1 / 2} .
$$

For the remainder of the paper, $\underline{d}$ will denote the degree sequence of $G$ (in any order). A direct simplification of $\sigma^{2}$ above shows that it is equal to $\frac{1}{(n-1)^{2}} S(m, n, p, \underline{d})^{2}$.

Lemma 3.4: Let $G$ be connected and let $\hat{G} \in \mathcal{G}(G, p)$. Then, for $\ell_{1: N}$ the smallest randomly sampled eigenvalue of $\hat{G}$, we have

$$
\mathbb{E}\left[\ell_{1: N}\right] \geq \max \left\{0, \frac{2 m p-S(m, n, p, \underline{d}) \sqrt{N-1}}{n-1}\right\}
$$


Proof: Note that because any Laplacian is positive semi-definite, $\mathbb{E}\left[\ell_{1: N}\right] \geq 0$. Then we apply Lemma 3.2 with $k=1$ to the values found in Lemma 3.3 to find that

$$
\begin{aligned}
\mathbb{E}\left[\ell_{1: N}\right] & \geq \frac{2 m p}{n-1}-\sigma \sqrt{\frac{N-1}{1}} . \\
& =\frac{1}{n-1}(2 m p-S(m, n, p, \underline{d}) \sqrt{N-1}) .
\end{aligned}
$$

Next, we leverage this bound on $\ell_{1: N}$ to bound the expectation of $\lambda_{2}$.

Lemma 3.5: Let $G$ be connected and let $\hat{G} \in \mathcal{G}(G, p)$, where $\hat{G}$ has Laplacian $L$. For $\lambda_{2}:=\lambda_{2}(L)$, we have

$$
\mathbb{E}\left[\lambda_{2}\right] \geq \max \left\{0,\left(1-\left(\frac{n-2}{n-1}\right)^{N-1}\right)^{-1}\left[\frac{2 m p}{n-1}\left(1-\left(\frac{n-2}{n-1}\right)^{N-1}\right)-\frac{1}{n-1} S(m, n, p, \underline{d}) \sqrt{N-1}\right]\right\} .
$$

Proof: We put Lemma 3.1 and Lemma 3.4 together to find that

$$
\mathbb{E}\left[\lambda_{2}\right]\left(1-\left(\frac{n-2}{n-1}\right)^{N-1}\right)+\frac{2 m p}{n-2}\left(\frac{n-2}{n-1}\right)^{N} \geq \frac{1}{n-1}(2 m p-S(m, n, p, \underline{d}) \sqrt{N-1})
$$

and solve this for $\mathbb{E}\left[\lambda_{2}\right]$.

We will also require a bound on $\lambda_{2}\left(L^{2}\right)$, which we present next.

Lemma 3.6: Let $G$ be connected and let $\hat{G} \in \mathcal{G}(G, p)$ have Laplacian $L$. Then

$$
\mathbb{E}\left[\lambda_{2}\left(L^{2}\right)\right] \leq \frac{1}{n-1}\left(4 m p-2 m p^{2}+p^{2} \sum_{i=1}^{n} d_{i}^{2}\right) .
$$

Proof: By definition, we have $\lambda_{2}\left(L^{2}\right) \leq \ell_{k}^{2}$ for an arbitrary $k$. Then

$$
\begin{aligned}
\mathbb{E}\left[\lambda_{2}\left(L^{2}\right)\right] & \leq \frac{1}{n-1} \operatorname{tr} \mathbb{E}\left[L^{2}\right] \\
& =\frac{1}{n-1} \sum_{i=1}^{n} \mathbb{E}\left[\left(L^{2}\right)_{i i}\right] \\
& =\frac{1}{n-1} \sum_{i=1}^{n} p d_{i}+d_{i}\left(d_{i}-1\right) p^{2}+p d_{i},
\end{aligned}
$$

which follows from Equation (7). Combining like terms, we find that

$$
\begin{aligned}
\mathbb{E}\left[\lambda_{2}\left(L^{2}\right)\right] & \leq \frac{1}{n-1}\left(\left(2 p \sum_{i=1}^{n} d_{i}\right)+\left(p^{2} \sum_{i=1}^{n} d_{i}^{2}\right)-\left(p^{2} \sum_{i=1}^{n} d_{i}\right)\right) \\
& =\frac{1}{n-1}\left(4 m p-2 m p^{2}+p^{2} \sum_{i=1}^{n} d_{i}^{2}\right),
\end{aligned}
$$

as desired.

With these basic relationships involving $\lambda_{2}$ now established, the next section presents the solution to Problem 1

\section{PRobabilistic Connectivity of Random Graphs and Unions of Random GRAPHS}

This section translates the bounds on $\ell_{1: N}$ derived in Section III for single random graphs into bounds on $\lambda_{2}$ for random graphs and unions of random graphs. We then present our solution to Problem 1 In this section, we use the notation $R(N, n)=1-((n-2) /(n-1))^{N-1}$. 


\section{A. Solution to Problem 1}

Toward solving Problem 1, we state the Paley-Zygmund inequality.

Lemma 4.1 (Paley-Zygmund inequality, [32]): Let $Z$ be a non-negative random variable with $\operatorname{Var}[\mathrm{Z}]<\infty$ and let $\theta \in[0,1]$. Then

$$
\mathbb{P}[Z>\theta \mathbb{E}[Z]] \geq(1-\theta)^{2} \frac{\mathbb{E}[Z]^{2}}{\mathbb{E}\left[Z^{2}\right]}
$$

We now present the main results of the paper, namely a solution to Problem 1 .

Theorem 4.2: (Solution to Problem 1) Let a connected graph $G$ on $n$ nodes be given, along with $p \in(0,1)$. Let $\hat{G} \in \mathcal{G}(G, p)$ and let $L$ denote its Laplacian with second-smallest eigenvalue $\lambda_{2}$. Then

$$
\mathbb{P}\left[\lambda_{2}>0\right] \geq \max _{N \in \mathbb{N}} \frac{(\max \{0,2 m p R(N, n)-S(m, n, p, \underline{d}) \sqrt{N-1}\})^{2}}{(n-1) R(N, n)^{2}\left(4 m p-2 m p^{2}+p^{2} \sum_{i=1}^{n} d_{i}^{2}\right)} .
$$

Proof: We apply Lemma 4.1 with $\theta=0$ using the estimates for $\mathbb{E}\left[\lambda_{2}\right]$ from Lemma 3.5 and $\mathbb{E}\left[\lambda_{2}\left(L^{2}\right)\right]$ from Lemma 3.6. Because Equation (8) holds for all $N \in \mathbb{N}$, it holds in particular for the maximum over $N$.

Maximizing over $N$ is finitely terminating, as shown in the following remark.

Remark 4.3: Observe that for a given $\mathcal{G}(G, p)$, the parameter $N \in \mathbb{N}$ must satisfy

$$
1 \leq N \leq \frac{2 m p(n-1)(2-p)+p^{2}(n-1) \sum_{i=1}^{n} d_{i}^{2}}{2 m p(n-1)(2-p)+p^{2}(n-1) \sum_{i=1}^{n} d_{i}^{2}-4 m^{2} p^{2}}
$$

to attain a positive value in the numerator of Equation (8). Thus the maximum occurs over this range of $N$, and, because this range is finite, searching for the maximum is finitely terminating.

Let $\hat{p}(T):=1-(1-p)^{T}$. We can then apply Theorem 4.2 to graph unions in terms of the number of time steps required to attain connectivity.

Theorem 4.4: Given $\epsilon>0$, the graph $\hat{G} \in \mathcal{U}_{T}(G, \hat{p}(T))$ is connected with probability $1-\epsilon$ after $T$ time steps if $T \geq T^{*}$, where $T^{*}$ is given by

$$
T^{*}=\min \left\{T \in \mathbb{N}: \max _{N \in \mathbb{N}} \frac{[\max \{0,2 m \hat{p}(T) R(N, n)-S(m, n, \hat{p}(T), \underline{d}) \sqrt{N-1}\}]^{2}}{(n-1) R(N, n)^{2}\left(4 m \hat{p}(T)-2 m \hat{p}(T)^{2}+\hat{p}(T)^{2} \sum_{i=1}^{n} d_{i}^{2}\right)} \geq 1-\epsilon\right\} .
$$

Proof: Using Lemma 2.5, this follows from Theorem 4.2 by replacing $p$ with $\hat{p}(T)$.

Remark 1: Examining Theorem 4.2 and Theorem 4.4, we see that the probability of connectedness is monotonically increasing in both $p$ and $T$. Thus, if agents in a network wish to increase the probability of connectedness of a union, they can either increase $p$, e.g., by spending more energy on communications, or wait for $T$ to increase as time elapses. To quantify this relationship, recall from Lemma 2.5 that, in a union of $T$ random graphs each with edge probability $p$, a single edge is present with probability $\hat{p}(T)=1-(1-p)^{T}$. Thus, regardless of the value of $p \in(0,1)$, there is some period of time $T$ over which the probability of an edge appearing can be made arbitrarily close to 1 . Consequently, varying $T$ can drive the probability of connectivity arbitrarily close to 1 as well, regardless of the probability of connectivity of any single graph in the union.

In the next section, we consider several specific families of graphs and compute explicit lower bounds for the solution to Problem 1 for these families as $n$ and $p$ range across several orders of magnitude. 


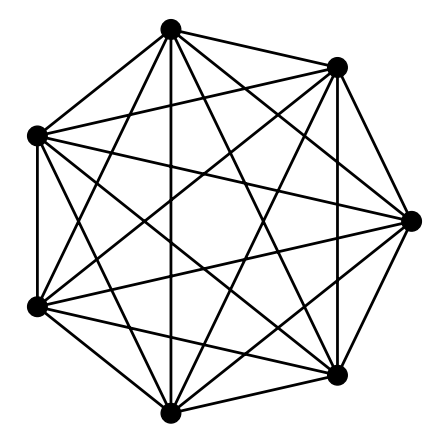

Fig. 2. Complete graph on 7 vertices, $K_{7}$

\section{Specific GRAPH Results}

This section illustrates our main results for two classes of underlying graphs: complete graphs and complete graphs minus a cycle.

\section{A. Complete Graph}

We consider random graphs $\hat{G} \in \mathcal{G}\left(K_{n}, p\right)$, where $K_{n}$ is the complete graph on $n$ nodes and $p \in(0,1)$. We note that this is equivalent to considering conventional Erdős-Rényi random graphs and these results therefore may be of independent interest.

Theorem 5.1: Let $K_{n}$ denote the complete graph on $n$ vertices and let $\hat{G} \in \mathcal{G}\left(K_{n}, p\right)$ have Laplacian $L$ with second-smallest eigenvalue $\lambda_{2}$. Then

$$
\mathbb{P}\left[\lambda_{2}>0\right] \geq \max _{N \in \mathbb{N}} \frac{\max \{0, \sqrt{n(n-1) p} R(N, n)-\sqrt{2(n-1)(1-p)(N-1)}\}^{2}}{(n-1) R(N, n)^{2}(2-2 p+n p)} .
$$

Proof: We apply Theorem 4.2 with $d_{i}=n-1$ for all $i$ and $m=\frac{n(n-1)}{2}$. Simplfying and factoring completes the proof.

Similar to Theorem 4.4, we have the following corollary for unions of graphs in $\mathcal{G}\left(K_{n}, p\right)$.

Theorem 5.2: Let $\hat{p}:=\hat{p}(T)$. Given $\epsilon>0$, a graph $\hat{U} \in \mathcal{U}_{T}\left(K_{n}, \hat{p}(T)\right)$ is connected with probability $1-\epsilon$ after $T$ time steps if $T \geq T^{*}$, where $T^{*}$ is given by

$$
T^{*}=\min \left\{T \in \mathbb{N}: \max _{N \in \mathbb{N}} \frac{\max \{0, \sqrt{n(n-1) \hat{p}} R(N, n)-\sqrt{2(n-1)(1-\hat{p})(N-1)}\}^{2}}{(n-1) R(N, n)^{2}(2-2 \hat{p}+n \hat{p})} \geq 1-\epsilon\right\} .
$$

We next examine numerical results. Figure 3 shows the probability of connectedness for a single random graph for $1 \leq n \leq 10,000$ and $0.8 \leq p \leq 1$. We see that increasing $n$ provides a modest increase in the probability of connectivity, while increasing $p$ makes connectivity substantially more likely. In addition, as noted in Remark 1 . accumulating more graphs in a union also increases the probability of connectivity. In particular, even if $p$ is small, a large $T$ can make $\hat{p}(T)$ large, thereby driving the probability of connectedness up according to the increases in probabilities seen in Figure 3 


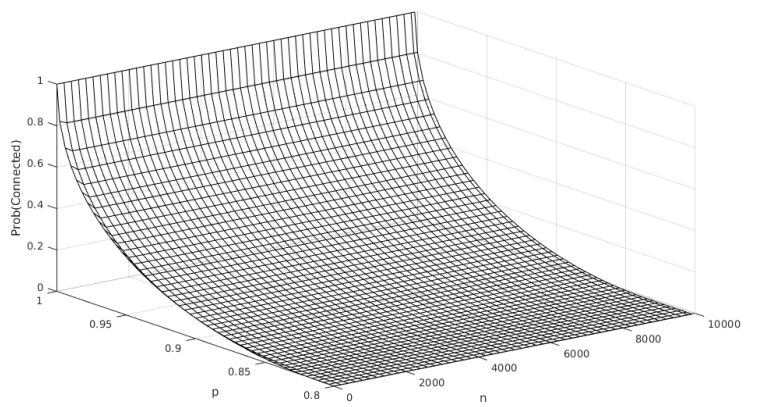

Fig. 3. Probability of connectedness of $\mathcal{G}\left(K_{n}, p\right)$ as a function of $n$ and $p$. As noted in Remark 1 the desired probability of connectedness can be attained for edge probabilities equal to the depicted values of $p$, or by waiting for $T$ to be large enough that the value of $\hat{p}(T)=1-(1-p)^{T}$ is equal to the desired depicted value of $p$.

\section{B. Complete Graph Minus a Cycle}

To illustrate our results beyond conventional Erdős-Rényi graphs, we now consider random graphs in which some edges never appear. Formally, define $K_{n} \backslash C_{n}$ to be the complete graph on $n$ vertices less the edges of a cycle on $n$ vertices. Then we consider random graphs $\hat{G} \in \mathcal{G}\left(K_{n} \backslash C_{n}, p\right)$.

Figure 5 shows the probability that a single graph $\hat{G} \in \mathcal{G}\left(K_{n} \backslash C_{n}, p\right)$ is connected for $1 \leq n \leq 10,000$ and $0.8 \leq p \leq 1$. We find that, for a fixed pair $(n, p)$, the probability of connectivity is less than it is for the same $(n, p)$ in Figure 3 . This is intuitive, as a graph in $\mathcal{G}\left(K_{n} \backslash C_{n}, p\right)$ has fewer ways to attain connectivity than a graph in $\mathcal{G}\left(K_{n}, p\right)$.

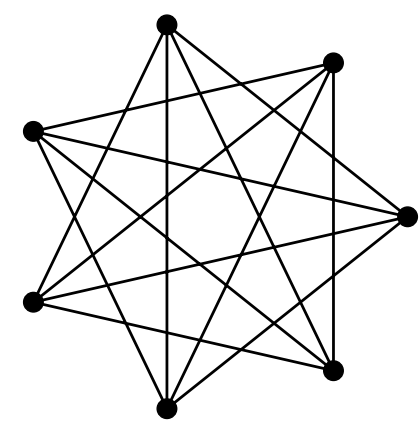

Fig. 4. Complete graph on 7 vertices with one cycle removed, $K_{7} \backslash C_{7}$.

\section{CONCLUSION}

We presented results bounding the probability of connectivity for random graphs and unions thereof. These results can be applied to multi-agent applications in both control and optimization, where some convergence rates explicitly depend upon the time needed to attain a connected union graph. Future work includes extensions to time-varying probabilities and heterogeneous edge probabilities. 


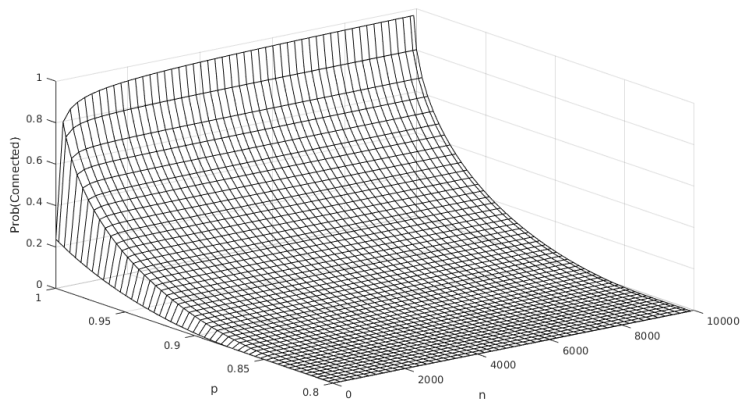

Fig. 5. Probability of connectedness of $\mathcal{G}\left(K_{n} \backslash C_{n}, p\right)$. Here, as in Figure 3 making $p$ larger for a single graph (or making $T$ larger to drive up $\hat{p}(T)$ for a union of graphs) increases the probability of connectivity.

\section{REFERENCES}

[1] Barry C. Arnold and Richard A. Groeneveld. Bounds on expectations of linear systematic statistics based on dependent samples. Ann. Statist., 7(1):220-223, 011979.

[2] Itai Benjamini, Simi Haber, Michael Krivelevich, and Eyal Lubetzky. The isoperimetric constant of the random graph process. Random Structures \& Algorithms, 32(1):101-114, 2008.

[3] V. D. Blondel, J. M. Hendrickx, A. Olshevsky, and J. N. Tsitsiklis. Convergence in multiagent coordination, consensus, and flocking. In Proceedings of the 44th IEEE Conference on Decision and Control, pages 2996-3000, Dec 2005.

[4] B. Bollobás. Random graphs. In Modern Graph Theory, pages 215-252. Springer, 1998.

[5] B. Bollobás. Random Graphs. Cambridge Studies in Advanced Mathematics. Cambridge University Press, 2nd edition, 2001.

[6] S. Caron and G. Kesidis. Incentive-based energy consumption scheduling algorithms for the smart grid. In First IEEE International Conference on Smart Grid Communications (SmartGridComm), pages 391-396, Oct 2010.

[7] Jeff Cheeger. A lower bound for the smallest eigenvalue of the laplacian. In Proceedings of the Princeton conference in honor of Professor S. Bochner, pages 195-199, 1969.

[8] A. Chen and A. Ozdaglar. A fast distributed proximal-gradient method. In 50th Annual Allerton Conference on Communication, Control, and Computing (Allerton), pages 601-608, 2012.

[9] A. Coja-Oghlan. On the Laplacian eigenvalues of G(n, p). Combinatorics, Probability, and Computing, 16(6):923-946, November 2007.

[10] Colin Cooper and Alan Frieze. The cover time of sparse random graphs. Random Structures \& Algorithms, 30(1-2):1-16, 2007.

[11] J. Cortes, S. Martinez, T. Karatas, and F. Bullo. Coverage control for mobile sensing networks. In International Conference on Robotics and Automation (ICRA), volume 2, pages 1327-1332, 2002.

[12] P. Diaconis and M. Shahshahani. On the eigenvalues of random matrices. Journal of Applied Probability, 31:49-62, 1994.

[13] P. Erdős and A. Rényi. On random graphs I. Publicationes Mathematicae Debrecen, 6:290-297, 1959.

[14] P. Erdôs and A Rényi. On the evolution of random graphs. In Publication of the Mathematical Institute of the Hungarian Academy of Sciences, pages 17-61, 1960.

[15] H. R. Feyzmahdavian and M. Johansson. On the convergence rates of asynchronous iterations. In 53rd IEEE Conference on Decision and Control, pages 153-159, Dec 2014.

[16] M. Fiedler. Algebraic connectivity of graphs. Czechoslovak Mathematical Journal, 23(2):298-305, 1973.

[17] Z. Füredi and J. Komlós. The eigenvalues of random symmetric matrices. Combinatorica, 1(3):233-241, 1981.

[18] C. Godsil and G. Royle. Algebraic graph theory, volume 207 of Graduate Texts in Mathematics. Springer-Verlag, New York, 2001.

[19] Y. Hatano and M. Mesbahi. Agreement over random networks. IEEE Transactions on Automatic Control, 50(11):1867-1872, Nov 2005.

[20] A. Jadbabaie, J. Lin, and A. S. Morse. Coordination of groups of mobile autonomous agents using nearest neighbor rules. IEEE Transactions on Automatic Control, 48(6):988-1001, June 2003.

[21] F. Kelly, A. Maulloo, and D. Tan. Rate control for communication networks: shadow prices, proportional fairness and stability. Journal of the Operational Research society, 49(3):237-252, 1998. 
[22] S. Kia, J. Cortés, and S. Martínez. Distributed event-triggered communication for dynamic average consensus in networked systems. Automatica, 59(C):112-119, September 2015.

[23] M. Krivelevich and B. Sudakov. The largest eigenvalue of sparse random graphs. Combinatorics, Probability, and Computing, 12(1):61-72, 2003.

[24] F. Lewis, H. Zhang, K. Hengster-Movric, and A. Das. Cooperative control of multi-agent systems: optimal and adaptive design approaches. Springer Science \& Business Media, 2013.

[25] I. Lobel and A. Ozdaglar. Distributed subgradient methods for convex optimization over random networks. IEEE Transactions on Automatic Control, 56(6):1291-1306, June 2011.

[26] M. Mesbahi and M. Egerstedt. Graph theoretic methods in multiagent networks. Princeton University Press, 2010.

[27] L. Moreau. Stability of multiagent systems with time-dependent communication links. IEEE Transactions on Automatic Control, 50(2):169182, Feb 2005.

[28] A. Nedic and A. Ozdaglar. On the rate of convergence of distributed subgradient methods for multi-agent optimization. In Proceedings of the 46th IEEE Conference on Decision and Control, pages 4711-4716, Dec 2007.

[29] A. Nedic and A. Ozdaglar. Distributed subgradient methods for multi-agent optimization. IEEE Transactions on Automatic Control, 54(1):48-61, Jan 2009.

[30] A. Nedic, A. Ozdaglar, and P. A. Parrilo. Constrained consensus and optimization in multi-agent networks. IEEE Transactions on Automatic Control, 55(4):922-938, April 2010.

[31] A. Olshevsky and J. N. Tsitsiklis. Convergence speed in distributed consensus and averaging. SIAM Review, 53(4):747-772, 2011.

[32] R. E. A. C. Paley and A. Zygmund. A note on analytic functions in the unit circle. Mathematical Proceedings of the Cambridge Philosophical Society, 28(3):266-272, 1932.

[33] Mohammad Pirani and Shreyas Sundaram. On the smallest eigenvalue of grounded laplacian matrices. IEEE Transactions on Automatic Control, 61(2):509-514, 2015.

[34] W. Ren and R. Beard. Consensus seeking in multiagent systems under dynamically changing interaction topologies. IEEE Transactions on Automatic Control, 50(5):655-661, May 2005.

[35] W. Ren, R. Beard, and T. McLain. Coordination variables and consensus building in multiple vehicle systems. In V. Kumar, N. Leonard, and A. Morse, editors, Cooperative Control: A Post-Workshop Volume, 2003 Block Island Workshop on Cooperative Control, pages 171-188. Springer, Berlin, Heidelberg, 2005.

[36] B. Touri and A. Nedic. Distributed consensus over network with noisy links. In 12th International Conference on Information Fusion, pages 146-154, July 2009.

[37] B. Touri and A. Nedic. On ergodicity, infinite flow, and consensus in random models. IEEE Transactions on Automatic Control, 56(7):15931605, July 2011.

[38] L. Tran, V. Vu, and K. Wang. Sparse random graphs: Eigenvalues and eigenvectors. Random Structures \& Algorithms, 42(1):110-134, 2013.

[39] P. Tseng. On the rate of convergence of a partially asynchronous gradient projection algorithm. SIAM Journal on Optimization, 1(4):603619, 1991.

[40] P. Tseng, D. P. Bertsekas, and J. N. Tsitsiklis. Partially asynchronous, parallel algorithms for network flow and other problems. SIAM Journal on Control and Optimization, 28(3):678-710, 1990.

[41] D. Watts and S. Strogatz. Collective dynamics of small-world networks. Nature, 393(6684):440-442, 1998.

[42] E. Wigner. On the distribution of the roots of certain symmetric matrices. Annals of Mathematics, 67(2):325-327, 1958.

[43] A. Yazicioglu, M. Egerstedt, and J. Shamma. Formation of robust multi-agent networks through self-organizing random regular graphs. IEEE Transactions on Network Science and Engineering, 2(4):139-151, October 2015.

[44] M. Zhu and S. Martinez. On distributed convex optimization under inequality and equality constraints. IEEE Transactions on Automatic Control, 57(1):151-164, Jan 2012.

\section{ACKNOWLEDGEMENTS}

The authors would like to thank the following funding sources for enabling this research: the cooperative agreement FA8650-19-2-2429 with USRA (Unviersities Space Research Association); the STRESS (Science, Technology 
and Research for Exploiting Sensor Systems) contract (FA8650-18-2-1645); Air Force Office of Scientific Research grant FA9550-19-1-0169; US Department of Defense's Science, Mathematics and Research for Transformation (SMART) Scholarship for Service Program; and the Air Force Research Lab Sensors Directorate. 